\title{
Peroxodisulfate reduction as a probe to interfacial charge
}

Ricardo Martínez-Hincapié, Víctor Climent, Juan M. Feliu*.

Instituto de Electroquímica and Departamento de Química Física. Universidad de Alicante. Apartado 99. E-03080 Alicante. Spain.

Abstract

Peroxodisulfate reduction has been investigated on $\mathrm{Pt}(111)$ on a broad range of $\mathrm{pH}$ values as a way to test interfacial charge. The reaction always proceeds in a potential window that is almost $\mathrm{pH}$ independent, regardless changes in the presence of adsorbed species. Comparison with other available information for these interphases allows to conclude that this reaction is very sensitive to the free charge on the metal, being inhibited when the latter is negative. This explains the complete current inhibition when the potential is lower than the potencial of zero free charge. Most remarkably, the inhibition at high potentials can be related with the existence of a second potential of zero charge, above which the surface becomes again negatively charged. This conclusion agrees with classical results for polycrystalline platinum (which has been reexamined), results from laser induced T-jump experiments and recent theoretical models of the interphase. In this way, this reaction is postulated as a new probe to locate the values of the potential of zero free charge.

Keywords: potential of zero charge; free charge; peroxodisulfate reduction; nitrous oxide reduction; platinum single crystal electrode.

*Corresponding author: juan.feliu@ua.es 
Introduction

The potential of zero charge (pzc) is a pivotal parameter in interfacial electrochemistry [1]. For liquid or coinage metal electrodes, the determination of the pzc is carried out by means of electrocapillary curves or by looking for the minimum differential capacity, respectively, as predicted by Gouy-Chapman theory [2]. In metals that adsorb hydrogen, or under the presence of other charge transfer processes, the later methods are precluded. Moreover, in the platinum/electrolyte interface, two different potentials of zero charge should be defined: the potential of zero total charge (pztc) and the potential of zero free charge (pzfc), each one related to total (q) or free charge $(\sigma)$, respectively. The pzfc is equivalent to the pzc in metals that do not adsorb hydrogen and follow Gouy-Chapman model [3].

The CO charge displacement experiment [4] can be used to determine the pztc on Pt(111) electrodes [5] and, from it, the pzfc can be estimated, after some non-thermodynamics assumptions [6]. Also, the laser induced temperature jump technique measures the potential of maximum entropy (pme) of interphase formation. At the pme the disorder of water molecules is maximal and its value is close to the pzfc [7]. The coincidence between the values of pme, coming from laser induced temperature jump, and those of pzfc estimated from CO charge displacement is quite remarkable [8]. Further studies have shown that the pzfc of $\mathrm{Pt}(111)$ is independent of $\mathrm{pH}$, as the pme is in solutions with $\mathrm{pH}<7[9,10]$.

Kolotyrkina et. al (reference 69 in [3]), using radiotracers techniques showed the existence of the pzfc, for oxidized platinum electrodes, a result anticipated by Balashova et. al. using electrokinetic measures (Reference 66 in [3]). In this respect, laser T-jump experiments show an inversion of the response when the surface becomes oxidized, agreeing with the existence of a second change in the orientation of interfacial water [11]. All these data were used by Huang et al. to convincingly model this behavior [12]. In a completely different 
approach, the use of local probes like nitrous oxide $\left(\mathrm{N}_{2} \mathrm{O}\right)$ reduction has also been proposed as an effective way to investigate interfacial properties $[13,14]$. Assuming that $\mathrm{N}_{2} \mathrm{O}$ reduction requires the adsorption of a weakly adsorbable molecule, its peak potential would appear when the surface is either free of adsorbed hydrogen or $\mathrm{OH}$, the main adsorbates on platinum electrodes in absence of anion specific adsorption. Moreover, the peak potential for the reduction of this neutral molecular probe was used further to estimate the local pztc's (from terraces and steps) on high index platinum single crystals electrodes. A good agreement between the values found using this molecular probe and those from charge displacement was established [15].

Another proposed probe reaction is the reduction of peroxodisulfate (PDS) anion, which is highly sensitive to the surface structure in low index platinum single crystal electrodes [16]. On Pt(111) the reaction at $\mathrm{pH} 1$ takes place in the double layer region and is inhibited near to $0.36 \mathrm{~V}$ vs RHE, whereas at $\mathrm{pH} 3$ the reaction is inhibited around $0.40 \mathrm{~V}$ vs RHE. Originally, this inhibition was related to hydrogen adsorption or the strong polarization of water molecules for each $\mathrm{pH}$, respectively. On $\mathrm{Pt}(110)$ and $\mathrm{Pt}(100)$ at $\mathrm{pH} 1$, PDS reduction occurs at $0.18 \mathrm{~V}$ and $0.45 \mathrm{~V}$ vs RHE, both potential values being close to the respective pztc's [16].

In this paper we examine PDS reduction to gain further information on the properties of this anionic probe. Single crystal platinum electrodes in non-adsorbing supporting electrolyte solutions with different $\mathrm{pH}$ were used. Results will be compared with other available data, to better understand these electrochemical interfaces.

\section{Experimental}

Electrochemical cell, electrode pretreatments, and electrochemical workstation have been described elsewhere [9]. All working solutions were prepared with ultra-pure water (ELGA, 18.2 M $\Omega \mathrm{cm}$ ), concentrated perchloric acid (Merk, for analysis), sodium fluoride (Merk, 
suprapur), $\mathrm{K}_{2} \mathrm{~S}_{2} \mathrm{O}_{8}$ (Fluka, 99.0\%). Hydrogen (N50), Argon(N50) and $\mathrm{N}_{2} \mathrm{O}$ (N25) were also used all from Air Liquide. The $\mathrm{pH}$ of the different solutions was calculated from potential measurements of the RHE as previously reported [10].

Results and discussion

Figure 1a shows PDS and $\mathrm{N}_{2} \mathrm{O}$ reduction cyclic voltammograms on a well ordered Pt(111) electrode at $\mathrm{pH} 1$. Results agree with those previously published $[15,16]$. The hydrogen adsorption region can be considered unaltered in the presence of both surface probes. On the other hand, the $\mathrm{OH}$ adsorption region is altered in the presence of the anionic probe, likely adsorbed in these conditions, but unaltered in the case of $\mathrm{N}_{2} \mathrm{O}$. Close inspection reveals that the potential of maximum current for $\mathrm{N}_{2} \mathrm{O}$ reduction coincides with the potential zone in which the PDS reduction drops to zero (0.3 V SHE; 0.4 V RHE ). This behavior most likely reflects the different charge values of each probe, neutral for $\mathrm{N}_{2} \mathrm{O}$ and negative for PDS, and how they interact with the surface electrode charge. In this way, the drop in PDS reduction current can be identified with the point when the charge on the electrode changes from positive to negative. That behavior suggests that PDS reduction is a suitable process to study interfacial surface charge.

To check the latter hypothesis, PDS reduction was carried out in a wide $\mathrm{pH}$ range. Figures 1 b-e show PDS and $\mathrm{N}_{2} \mathrm{O}$ reduction in other different $\mathrm{pH}$ solutions, in the SHE scale, in which clear trends are observed: at pH 1 the PDS reduction starts and finishes in the double layer region; for $\mathrm{pH} 3$, the reduction begins in the $\mathrm{OH}$ adsorption region while the inhibition appears in the middle of the double layer region; finally, at $\mathrm{pH} 5$ the reaction occurs completely in the $\mathrm{OH}$ adsorption region. It is clear from this figure that onset and inhibition potentials are not governed by the presence of adsorbed species $(\mathrm{OH}$ or anion and hydrogen, respectively). It is remarkable that the potential window where PDS reaction takes place is the same for all pHs in the SHE scale. Furthermore, the potential where PDS reduction drops to 
zero coincides with the potential of maximum current for $\mathrm{N}_{2} \mathrm{O}$ reduction in the whole $\mathrm{pH}$ range studied. At this point, the sign of the charge on the electrode seems to be the main parameter governing PDS reduction instead of other factors (polarization of interfacial water molecules in pme determination or hydrogen/OH adsorption in $\mathrm{N}_{2} \mathrm{O}$ reduction). To evidence this point, subtraction of the blank current density from the current density for PDS reduction was performed. The current difference falls to zero at the same potential, $0.28 \pm 0.01 \mathrm{~V}$ vs SHE, in the whole $\mathrm{pH}$ range studied (Figure $2 \mathrm{~A}-\mathrm{B}$ ) and is practically independent of the sweep direction. This potential value, $\mathrm{E} @ \mathrm{j}=0$, is almost the same as the values reported for the pzfc or pme for the Pt(111) electrode. A feasible explanation of the inhibition of the PDS reduction is that at $\mathrm{E} @ \mathrm{j}=0$ the electrode surface charge changes from positive to negative. This transition hampers the adsorption and subsequent reaction of a negative species, such as the PDS probe. Contrarily, the current for the $\mathrm{N}_{2} \mathrm{O}$ reduction is maximum at the pzfc since it is a neutral molecule and its adsorption is less affected by the electrode charge. Complications arise from the fact that, unlike PDS reduction to sulphate, the thermodynamic potential for $\mathrm{N}_{2} \mathrm{O}$ reduction is $\mathrm{pH}$ dependent. Contrarily, PDS and sulphate adsorb on Pt(111) more strongly than $\mathrm{N}_{2} \mathrm{O}$ or its reduction products. Table 1 summarizes the values for the pzfc, pme and $\mathrm{E} @ j=0$ for all pHs studied here. For $\mathrm{Pt}(111)$ the values are very close and the slope of their change with $\mathrm{pH}$ is almost the same.

Another interesting point deals with the onset potential for PDS reduction. A nearly constant onset potential value is found, $0.72 \mathrm{~V}$ for all $\mathrm{pHs}$ (in SHE scale). Maintaining the same hypothesis postulated above, since the reduction of PDS requires a positive electrode charge and it is inhibited when this charge becomes negative, the reduction reaction would not start until the electrode charge becomes positive. This may indicate the existence of other transition point, where the charge on the electrode switches from negative to positive (when scanning from positive towards negative values). In other words, it probes the existence of a second potential of zero charge at high potentials, as proposed previously [3]. Garcia-Araez et. 
al. reported for $\mathrm{Pt}(111)$ electrode in a $\mathrm{pH}=3$ solution a similar non-monotonic behavior for the thermal coefficient of the double layer which is closely related with the water dipole orientation [11]. As mentioned, Huang et. al. proposed a double layer model, based on these experimental data, involving three charge regions on $\mathrm{Pt}(111)$ : a negative charge region at low potentials (hydrogen region) followed by a positive charge region at intermediate potentials and again a negative charge region ascribed to surface oxide dipoles [12]. The second transition was calculated to be around $0.7 \mathrm{~V}$ vs RHE at $\mathrm{pH} 1.2$. Remarkably, this potential is close to the onset potential for PDS reduction and indicates that PDS reduction could be used as a molecular probe to determine this second pzfc, pointed out in polycrystalline platinum by using a completely different method.

In order to check the possible agreement with such classical results, PDS reduction was studied on polycrystalline $\mathrm{Pt}$ after extensive potential cycling at $\mathrm{pH} 5.2$, a well buffered solution with pH close to that described by Frumkin and Petrii. Results are shown in figure 3 ab. It is clear that the number of peaks in the voltammogram reflects the local character of the PDS probe, a subject that should be the object of future studies. However, the interesting result is that the higher $\mathrm{E} @ \mathrm{j}=0.49 \mathrm{~V}$ vs $\mathrm{SHE}$, coincides with that reported in the classical paper by Frumkin and Petrii, $0.47 \mathrm{~V}$ vs NHE. Unfortunately, the contributions coming from PDS reduction on $\{100\}$ sites and, less important, $\{110\}$ sites, mask the decrease of current in the first minimum, which should generate the lower E@j=0. The PDS reduction signal, however, can be reasonably deconvoluted (Figure $3 b$ ) and then an estimation of the lower $E @ j=0$ can be made. Again, the second $E @ j=0$ value coming from the deconvolution, $0.15 \vee$ vs SHE, reasonably agree with the previously reported, $0.13 \mathrm{~V}$ vs NHE [3]. This experiment on polycrystalline surfaces also suggests that PDS reduction can be used as probe of the two pzfc values, taken into account the local contributions of the different sites. It is unclear why the results from Frumkin and Petrii approach better those at $\mathrm{Pt}(111)$ than those of the other basal plane orientations. Clearly, more systematic work should be made with stepped platinum 
surfaces to understand the validity of the use of this simple surface probe on polycrystalline oriented surfaces.

Conclusions

PDS reduction has been studied on $\mathrm{Pt}(111)$ in a wide $\mathrm{pH}$ range and compared with $\mathrm{N}_{2} \mathrm{O}$ reduction. PDS reduction is used as an inexpensive and effortless charged surface probe to estimate the pzfc on platinum single crystal electrodes. A good agreement between the values found here and the values coming from previous studies supports the validity of the use of PDS reduction to explore interfacial properties and, in this specific instance, the value of pzfc. The classic Frumkin hypothesis about the presence of two pzc is also explored. The onset potential of PDS reduction is used as indicative of the position of this second pzc. Moreover, comparison with values reported by Garcia-Araez et. al.[11] and Huang et. al. [12] reveals a good coincidence. The use of stepped surfaces could bring clues about the determination of local properties. Preliminary results suggest that the use of this charged surface probe can also be applied to this purpose.

\section{References}

1. Frumkin, A., O. Petrii, and B. Damaskin, The notion of the electrode charge and the Lippmann equation. Journal of Electroanalytical Chemistry and Interfacial Electrochemistry, 1970. 27(1): p. 81-100.

2. Silva, F., M.J. Sottomayor, and A. Hamelin, The temperature coefficient of the potential of zero charge of the gold single-crystal electrode/aqueous solution interface: Possible relevance to gold-water interactions. Journal of Electroanalytical Chemistry and Interfacial Electrochemistry, 1990. 294(1): p. 239-251.

3. Frumkin, A.N. and O.A. Petrii, Potentials of zero total and zero free charge of platinum group metals. Electrochimica Acta, 1975. 20(5): p. 347-359.

4. Clavilier, J., et al., Study of the charge displacement at constant potential during $\mathrm{CO}$ adsorption on Pt(110) and Pt(111) electrodes in contact with a perchloric acid solution. Journal of Electroanalytical Chemistry, 1992. 330(1): p. 489-497.

5. Feliu, J.M., et al., New information on the unusual adsorption states of Pt(111) in sulphuric acid solutions from potentiostatic adsorbate replacement by $\mathrm{CO}$. Journal of Electroanalytical Chemistry, 1994. 372(1): p. 265-268.

6. Weaver, M.J., Potentials of Zero Charge for Platinum(111)-Aqueous Interfaces: A Combined Assessment from In-Situ and Ultrahigh-Vacuum Measurements. Langmuir, 1998. 14(14): p. 3932-3936. 
7. Climent, V., B.A. Coles, and R.G. Compton, Coulostatic Potential Transients Induced by Laser Heating of a Pt(111) Single-Crystal Electrode in Aqueous Acid Solutions. Rate of Hydrogen Adsorption and Potential of Maximum Entropy. The Journal of Physical Chemistry B, 2002. 106(23): p. 5988-5996.

8. Sebastián, P., et al., Study of the Pt (111) / electrolyte interface in the region close to neutral $\mathrm{pH}$ solutions by the laser induced temperature jump technique. Electrochimica Acta, 2017. 228(Supplement C): p. 667-676.

9. Martínez-Hincapié, R., et al., Exploring the interfacial neutral pH region of Pt(111) electrodes. Electrochemistry Communications, 2015. 58(Supplement C): p. 62-64.

10. Rizo, R., et al., Towards the understanding of the interfacial $\mathrm{pH}$ scale at Pt(111) electrodes. Electrochimica Acta, 2015. 162(Supplement C): p. 138-145.

11. Garcia-Araez, N., V. Climent, and J. Feliu, Potential-Dependent Water Orientation on Pt(111), Pt(100), and Pt(110), As Inferred from Laser-Pulsed Experiments. Electrostatic and Chemical Effects. The Journal of Physical Chemistry C, 2009. 113(21): p. 92909304.

12. Huang, J., et al., Non-monotonic Surface Charging Behavior of Platinum: A Paradigm Change. The Journal of Physical Chemistry C, 2016. 120(25): p. 13587-13595.

13. Ebert, H., et al., The reduction of nitrous oxide on platinum electrodes in acid solution. Journal of Electroanalytical Chemistry and Interfacial Electrochemistry, 1989. 264(1): p. 181-193.

14. Attard, G.A. and A. Ahmadi, Anion-surface interactions Part 3. N2O reduction as a chemical probe of the local potential of zero total charge. Journal of Electroanalytical Chemistry, 1995. 389(1): p. 175-190.

15. Climent, V., G.A. Attard, and J.M. Feliu, Potential of zero charge of platinum stepped surfaces: a combined approach of $\mathrm{CO}$ charge displacement and $\mathrm{N} 2 \mathrm{O}$ reduction. Journal of Electroanalytical Chemistry, 2002. 532(1): p. 67-74.

16. Climent, V., et al., Peroxodisulphate reduction as a novel probe for the study of platinum single crystal/solution interphases. Journal of Electroanalytical Chemistry, 2008. 612(2): p. 269-276. 


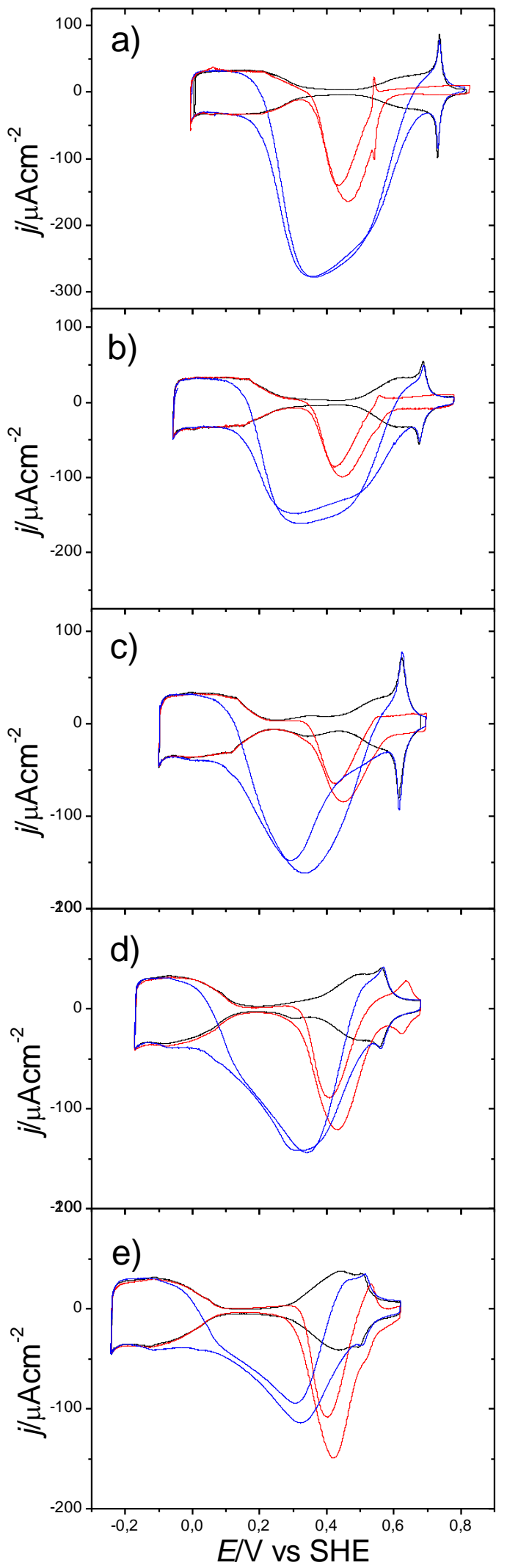

Figure 1. PDS (red) and $\mathrm{N}_{2} \mathrm{O}$ (blue) reduction on Pt(111) in pH 1.1 (a), 2.1 (b), 3.0 (c), 4.1 (d) and 5.0 (e). Scan rate $50 \mathrm{mVs}^{-1}$. The blank voltammograms are also showed (black). 


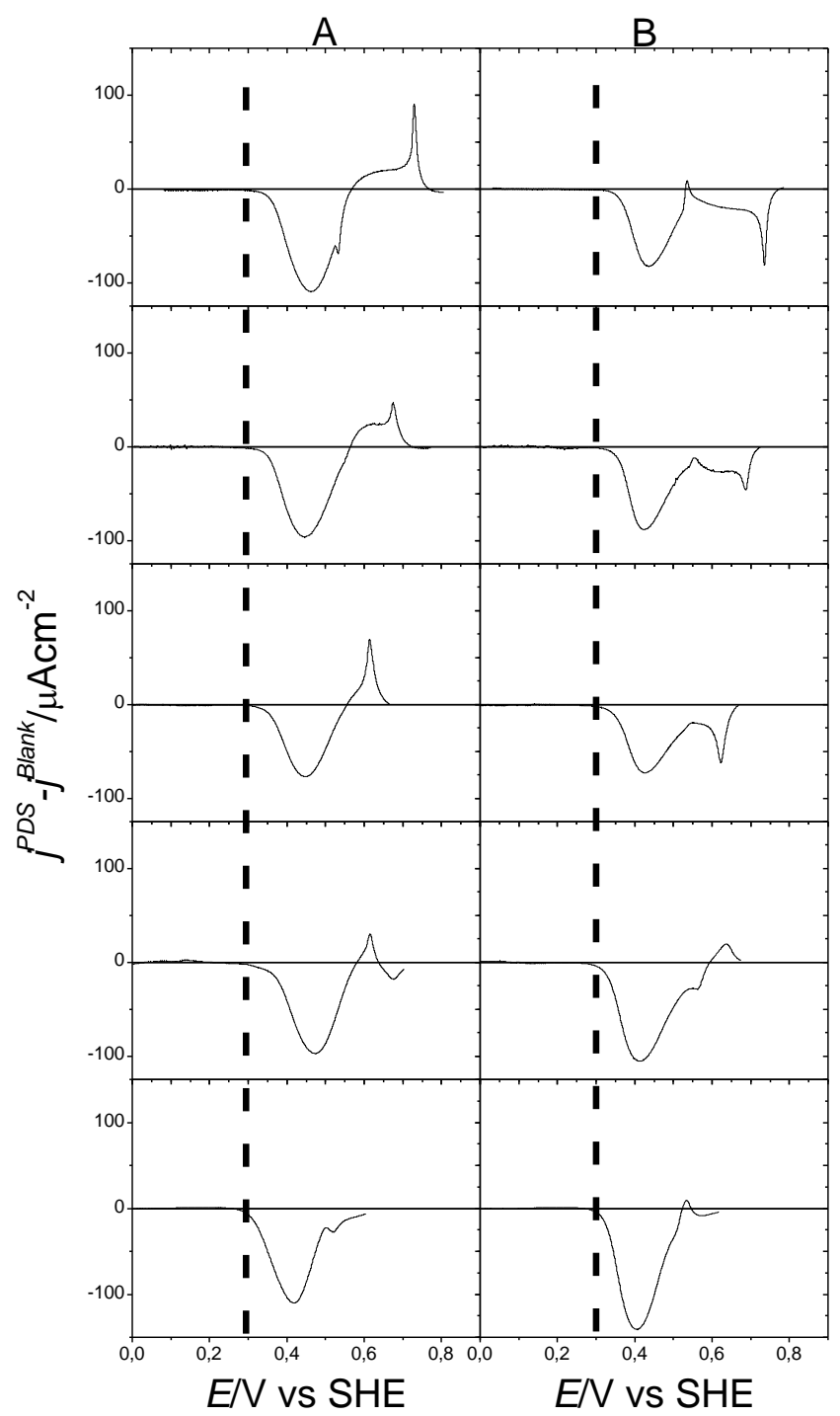

Figure 2. Subtraction of the blank current density $\left({ }^{\text {blank }}\right)$ to the current density for PDS reduction $\left({ }^{\text {PDS }}\right)$. A) Negative and B) positive going-sweeps. Same conditions as in figure 1.

\begin{tabular}{|l|l|l|l|}
\hline $\mathrm{pH}$ & $\mathrm{E} @ \mathrm{j}=0$ & $\operatorname{pzfc}[9,10]$ & $p m e[8,11]$ \\
\hline 1.1 & 0.292 & 0.300 & 0.300 \\
\hline 2.1 & 0.294 & 0.320 & 0.312 \\
\hline 3.0 & 0.283 & 0.282 & 0.300 \\
\hline 4.1 & 0.270 & 0.285 & 0.320 \\
\hline 5.0 & 0.270 & 0.272 & 0.300 \\
\hline
\end{tabular}

Table 1. Values of E@j=0, pzfc and pme. 


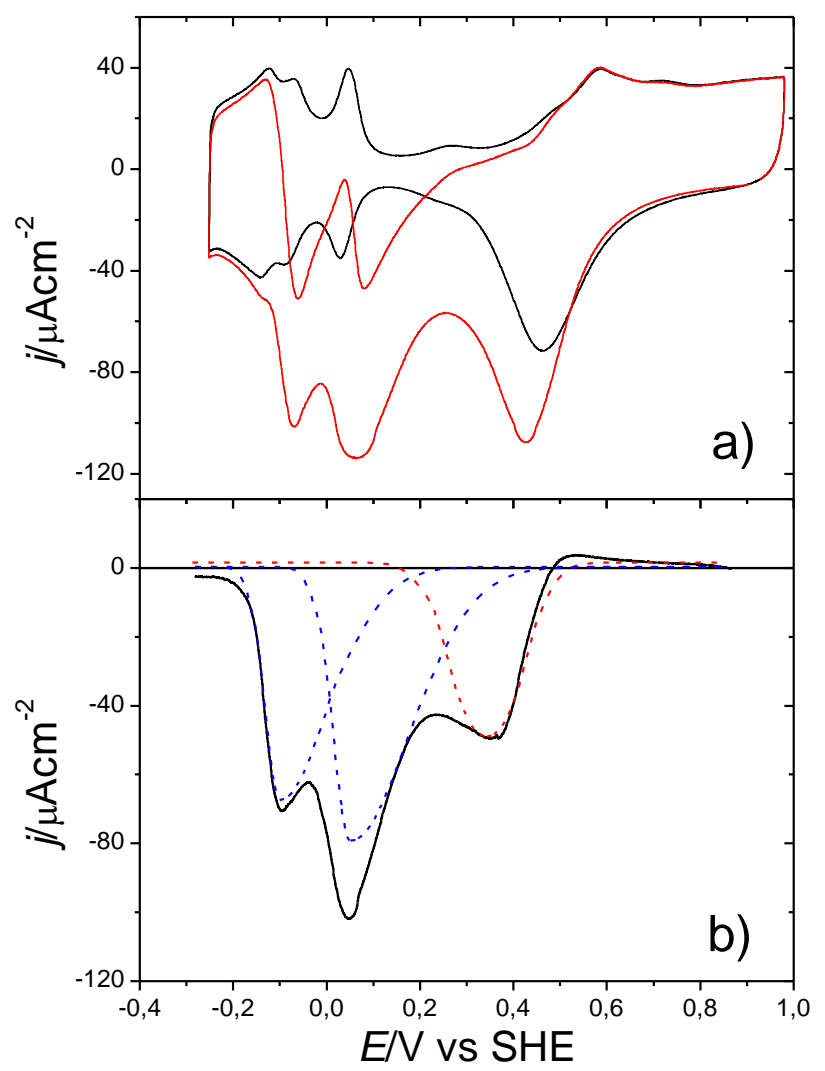

Figure 3. a) PDS reduction on polycrystalline Pt at pH 5.2 (red). The blank voltammogram is also showed (black). b) Subtraction of jblank to jPDS on the negative-sweep scan (black) and deconvoluted responses (dashed lines). Deconvolution was performed using a Gaussian/Bigaussian functions. Scan rate $50 \mathrm{mVs}^{-}{ }^{1}$. 